\title{
An Investigation into the Cognitive Deficits Associated with Chronic Fatigue Syndrome
}

\author{
Marie Thomas*,1,2 and Andrew Smith ${ }^{1}$ \\ ${ }^{I}$ Centre for Occupational and Health Psychology, School of Psychology, Cardiff University, UK \\ ${ }^{2}$ Centre for Health Information Research Evaluation, School of Medicine, Swansea University, UK
}

\begin{abstract}
This study addresses, among other things, the debate as to whether cognitive deficits do occur with a diagnosis of Chronic Fatigue Syndrome (CFS). Previous studies have indicated a potential mismatch between subjective patient ratings of impairment and clinical assessment. In an attempt to tackle some of the methodological problems faced by previous research in this field, this study recruited a large sample of CFS patients where adequate diagnosis had been made and administered an extensive battery of measures. In doing so this study was able to replicate previous published evidence of clear cognitive impairment in this group and demonstrate also that these deficits occurred independent of psychopathology. The conclusion drawn is that cognitive impairments can be identified if appropriate measures are used. Furthermore, the authors have shown that performance changes in these measures have been used to assess both efficacy of a treatment regime and rates of recovery.
\end{abstract}

Keywords: Chronic fatigue syndrome, cognitive deficits, healthy controls.

\section{INTRODUCTION}

Fatiguing illnesses, in particular Chronic Fatigue Syndrome (CFS), are difficult conditions to accurately diagnose and quantify. The Oxford [1] and Centre for Disease Control (CDC) [2] criteria for CFS define a person suffering from CFS as one who has experienced persistent, debilitating mental and physical fatigue for six months or more, where rest is not restorative and the fatigue state is not due to ongoing exertion. There may also be several co-existing symptoms present at any time, including those of a cognitive or neuropsychiatric nature, and once these are established, cognitive, behavioral, emotional, physiological and social factors are thought to work together to perpetuate it [3]. The clinical features of the illness include reports by patients of impaired cognition and research groups have investigated whether an association between mental fatigue and cognitive functioning occurs $[4,5]$. Evidence to suggest the existence of impairments in focused attention, speed of processing and performance accuracy in CFS are supported by data which indicate that as sleep deprived, healthy individuals become more fatigued, cognitive deficits become more pronounced [6].

The deficits associated with physical fatigue experienced by CFS sufferers have also been well documented over the past ten years. Decrements in performance on both simple and choice reaction time measures, for example, were indicted by LaManca et al. in 1998 [7] and studies have since revealed impairments in verbal fluency, memory, motor speed, sustained attention and speed of cognitive processing [8-12]. Others have highlighted deficits in verbal and nonverbal memory tasks occurring in this illness [13, 14].

*Address correspondence to this author at the Centre for Health Information Research Evaluation, School of Medicine, Swansea University, UK; E-mail: Marie.A.Thomas@swansea.ac.uk
Others have, however, questioned whether cognitive impairment does actually occur $[15,16]$, especially in the light of apparent discrepancies between objective measures of impairment and the subjective measures of cognitive failures reported by sufferers [17]. There is evidence to suggest that patient-rated perceived levels of impairment appear much higher than their performance on objective measures. Wearden and Appleby [18], although acknowledging this, indicated that the lack of conclusive data may more likely be explained by the fact that the tasks chosen to assess cognition were not of sufficient sensitivity to measure the impairments in CFS. They further proposed that the performance impairments reported were only evident in tests of a more complicated nature, such as tasks requiring the completion of two or more elements simultaneously (the dual-task paradigm). A recommendation made in light of this paper suggested that future research should employ measures that reflect the nature of the specific cognitive complaints patients themselves report.

Methodological considerations aside, factors such as sample demographics, intelligence, anxiety and depression are known to affect cognitive performance. The link between reaction time, age and intelligence, for example, has long since been established [19]. Similarly, mood disorders such as depression and anxiety are associated with memory and additional deficits [20-22]. In light of this, Michiels and Cluydts [23] expressed the concern that it may be the demographic nature of the patient sample and the presence of comorbid psychopathology that were responsible for any cognitive deficits reported and not the illness itself. To illustrate this several instances were highlighted where comparison groups had not been sufficiently matched to the CFS sample and screening for co-morbid anxiety and/or depression were not rigorously applied.

To further complicate this particular area of research, the heterogeneous nature of CFS is well documented [24] dictat- 
ing that any study investigating cognitive impairment in this illness would require large numbers of patients. Indeed, our previous studies had been conducted on relatively small patient numbers [25] and, although findings were encouraging, it was acknowledged that larger patient numbers were required for a more accurate profile of impairment to emerge. In addition, the analyses conducted would need to be extended to include, not only the potentially confounding factors that co-exist in the illness (such as age, gender and comorbid depression), but also mechanisms (such as the impact of stress, daily hassles and life events) which appear to exacerbate and perpetuate the condition.

In view of the on-going debate as to the existence and exact nature of the cognitive deficits associated with CFS the current study addressed each of the conflicting issues raised by: (a) recruiting a large sample of Chronic Fatigue Syndrome sufferers from a specialized out-patient clinic where strict adherence to the CDC criteria was maintained [2]; (b) comparing the patient group to an age-, gender- and educationally-matched healthy control group; (c) administering a wide range of objective and subjective measures developed from previous studies [8, 25]; and (d) identifying possible confounding factors which could account for any deficits observed and incorporate them into the analysis model.

\section{MATERIALS AND METHODS}

Ethical approval was granted by the local research ethics committee and informed consent was obtained from all who participated. Data were coded to protect the anonymity of both patient and control groups.

\section{Design}

Baseline data collected from the patient group were compared to data from a matched group of healthy controls in a between group design.

\section{Participants}

Patients, all of whom had been referred by their GP and were attending an hospital infectious diseases outpatient clinic specifically set up to research CFS, were invited to join a volunteer panel administered by the Health Psychology Research Unit. In order to be recruited to the research panel, a patient had to fulfill the Centre for Disease Control (CDC) criteria [2]. Each patient completed a comprehensive batch of computerized mood and cognitive performance tasks as well as a wide range of questionnaires including illness history, psychopathology (in this case co-morbid anxiety and depression), health and well-being. A healthy control group of 126 participants, consisting of members of the general population, was set up by advertisement in local newspapers. This control group provided appropriately age-, gender- and educational-status-matched comparisons for the Chronic Fatigue Syndrome sample. The testing session for both CFS patients and controls were conducted by psychology researchers with an average duration of forty minutes depending on the needs of each participant.

\section{QUESTIONNAIRES}

\section{Demographics and Illness History}

The demographic section of the questionnaire, as well as collecting data relating to gender, age, social status and educational background of the participants, required the CFS cational background of the participants, required the CFS patients to provide a brief history of their illness including illness length, GP involvement in diagnosis, type of onset, illness severity and illness characteristics [8]. The National Adult Reading Test (NART) [26] was used in this study to measure pre-morbid intelligence in the patient and control groups.

\section{Health-Related Behaviors}

Two variables, namely the average number of hours slept per night and a score rating 'feeling rested from sleep', were used to measure sleep quality in the sample. In addition, activity levels, alcohol and cigarette consumption and eating habits were also assessed, as were the use of prescribed medication and dietary supplements [8].

\section{Symptoms and Illness Severity}

Both patients and controls also completed a 28-item symptom check list [27], the total scores from which were also used as a measure of illness severity, as was the Profile of Fatigue Related Symptoms questionnaire (PFRS) [28].

\section{Psychopathology, Mood and Cognition}

The specific measures described above were considered in conjunction with subjective ratings of positive and negative affect [29], depression [30] and anxiety [31]. In addition, subjective measures of cognition were measured using the Cognitive Failures Questionnaire (CFQ) [32].

\section{Psychosocial Measures}

Measures of perceived stress [33], major life events [34], daily hassles [35] and self esteem [36] were used to investigate psychosocial factors and their influence on this illness.

\section{MOOD AND PERFORMANCE TESTING}

A battery of computerized performance tests were used to assess mood, memory, attention and psychomotor function [8]. Responses were registered using a response box connected to a timer card allowing measurement of reaction times to the nearest millisecond.

\section{Mood Scales}

Subjective mood was assessed using eighteen computerised visual analogue mood scales. Each of these bipolar scales comprized a pair of adjectives, for instance drowsy -alert or happy - sad. Participants were instructed to move the cursor from a central position on the scale to a location anywhere along the horizontal line until the cursor rested at a position which was representative of their current mood state. Three scores were derived using factor analysis from the original eighteen items: alertness, hedonic tone and anxiety.

\section{Free Recall}

This task assessed short-term recall. Volunteers were shown a list of 20 words presented at a rate of one every two seconds. At the end of the list the volunteer had two minutes to write down (in any order) as many of the words as possible on the sheet provided. The variables measured were the number of words written down, the number of correct words and the number of incorrect words. 


\section{Variable Fore-Period Simple Reaction Time Task (Three Minutes Duration)}

In this task a frame of a box was displayed in the centre of the screen and at varying intervals (from 1 to 8 seconds) a target square appeared inside the box (approximately 8 per minute). As soon as the participant detected the square, they were required to press the response key using the forefinger of their dominant hand only. A reaction time was measured for each presentation and a mean reaction time was calculated for each minute of performance on the basis of the number of trials completed per minute. An overall mean reaction time was calculated from the total number of trials completed over the duration of the task.

\section{Repeated Digits Vigilance Task (Three Minutes Dura- tion)}

This is a measure of vigilance where ability to detect targets at irregular intervals is assessed. Participants were shown successive presentations of three-digit numbers in the centre of the screen (e.g. 473) at the rate of 100 per minute. Each three-digit number usually differed from the one immediately preceding it, with one out of the three digits being replaced with a different digit (e.g. 463, 563, 562). Occasionally ( 8 times a minute) the same three-digit number was presented on successive trials. It was these repetitions that the participant needed to detect and respond as quickly as possible by pressing a key on the response box using the forefinger of their dominant hand. For each minute of the task and over the duration of the task measures of total mean reaction time to targets, total of trials correctly detected (hits) and the total number of false alarms were recorded.

\section{Distraction from Irrelevant Stimuli}

In addition to the computerised tests, the patients and controls carried out the Stroop colour-word interference task [37] which measured distraction from irrelevant stimuli. The task required the participant to complete two control and two test conditions which involved the identification of four colours; red, blue, green or yellow. Firstly, the subject was asked to read the word control card. This involved reading a series of words (red, blue, yellow or green) aloud from left to right and from top to bottom. Next, the participant completed the word test. Here the test card was covered with examples of the four colour words which were coloured differently to that word (that is, the word blue may be coloured yellow, etc.). The volunteer was instructed to read the words only. The third part of the task involved describing the colours presented on the control card (again, from left to right and from top to bottom). Each colour was represented as a series of dots. Finally, the volunteer was asked to repeat the test card; this time describing the colour of the word and not the word itself. Each section of the task was timed using a stopwatch. The times for the word or colour interference section of the task were calculated by subtracting the control time from the test time.

\section{PROCEDURE}

Two separate questionnaire booklets were administered in total; one was completed before visiting the clinic or research centre (including demographic data, illness history and symptom severity, health-related behaviours, psychopathology, psychosocial factors and measures of cognition), and one at the time of testing (administered before the com- pletion of the cognitive function tasks and including measures assessing the level of state anxiety, depression, negative and positive affect and fatigue-related symptoms within the previous week).

\section{Sample Size}

Priori power analysis [38] calculated an effect size of 0.98 for the simple reaction time task data from our previous study [25]. It was calculated from these data that a sample size of 24 CFS patients and 24 controls would have $95 \%$ chance of detecting an impairment effect at the $5 \%$ level of significance.

\section{Data Analysis}

The first set of analyses described and compared data from the two groups. Categoric data were analysed using Chi-squared tests and analyses of variance were used for continuous data. Repeated-measures analyses of variance were used to assess the impact of time on task.

Next, analyses of covariance were used to identify possible confounding factors for each of the four performance measures (short-term memory, motor speed, vigilance and cognition). Separate analyses were carried out using a range of factors including demographics, health-related behaviours, symptoms, psychopathology, mood and cognition, and psychosocial factors as covariates. Factors identified as those exerting an independent effect on each performance task were included in the analysis model to assess their role in the overall group effect. In addition, these factors (identified as confounds for each performance task) were used as covariates in subsequent analyses.

The final part of the study investigated whether subjective ratings of cognition and illness severity were linked to objective performance deficits within the CFS group as debated previously [17, 18]. Measures, including cognitive failures, fatigue-related measures and total symptom scores, were used as grouping factors for these analyses.

\section{RESULTS}

\section{The Chronic Fatigue Syndrome Patient Sample}

The CFS group recruited to this study had a mean illness length of five years and $68 \%$ of the patients had received a preliminary diagnosis of CFS by their general practitioner (GP). $84 \%$ of the patients believed that a specific event had preceded their illness, the majority of these respondents naming influenza as the event $(42 \%)$, but some $(25 \%) \mathrm{did}$ acknowledge that stress may have had a causative effect. In over $50 \%$ of the cases there was no fixed pattern in terms of symptom severity and time of day. The majority of the patients $(41 \%)$ rated illness status as 'bad with some recovery'. When asked if there was anything that improved their symptoms, $69 \%$ indicated that rest and $50 \%$ that sleep had a beneficial effect. Exercise, walking, shopping, mental effort and stress were highlighted as factors which exacerbate symptoms of the illness.

\section{CHRONIC FATIGUE SYNDROME AND CONTROL GROUP COMPARISONS}

\section{Demographic Data}

Table 1 describes basic demographic data for the CFS and control groups. The groups were very similar in terms of 
Table 1. The Demographic Data and Health-Related Measures for the CFS and Control Samples. Scores are the Group Means with s.e.m in Parenthesis, or Expressed as Percentages

\begin{tabular}{|c|c|c|c|}
\hline & CFS $(\mathrm{N}=307)$ & Controls $(\mathrm{N}=126)$ & $\mathrm{F}\left(\right.$ or $\left.\chi^{2}\right), \mathrm{df}, \mathrm{p}$ \\
\hline \multicolumn{4}{|l|}{ Demographic Data: } \\
\hline Male: Female (ratio) & $30: 70$ & $34: 66$ & $\mathrm{n} / \mathrm{s}$ \\
\hline Age (years) & $42.09(0.67)$ & $40.88(1.18)$ & $\mathrm{n} / \mathrm{s}$ \\
\hline Marital Status Married (\%) & 64.6 & 50.8 & $\mathrm{n} / \mathrm{s}$ \\
\hline Educational Status: Degree level (\%) & 17.3 & 16.7 & $\mathrm{n} / \mathrm{s}$ \\
\hline Employment Status: Employed (\%) & 32.4 & 50.0 & $24.34,5,0.001$ \\
\hline Social Classification: Skilled manual (\%) & 6.6 & 13.2 & $11.69,5,0.039$ \\
\hline $\boldsymbol{N A R T}$ (errors) & $14.72(0.51)$ & $12.48(0.71)$ & $5.93,1430,0.015$ \\
\hline \multicolumn{4}{|l|}{ Health-related Behaviours: } \\
\hline Medication (yes) & 61.3 & 27.0 & $41.80,1,0.001$ \\
\hline Multi-Vitamins (yes) & 51.0 & 23.0 & $28.43,1,0.001$ \\
\hline Average Sleep (hours) & $7.61(0.11)$ & $7.30(0.09)$ & $\mathrm{n} / \mathrm{s}$ \\
\hline Rested by Sleep (never) & 18 & 1 & $126.00,4,0.001$ \\
\hline Eating Well (yes) & 69.8 & 65.6 & $\mathrm{n} / \mathrm{s}$ \\
\hline Drink Alcohol (no) & 23.2 & 9.6 & $29.81,3,0.001$ \\
\hline Smoker (no) & 81.8 & 67.5 & $10.49,1,0.001$ \\
\hline Exercise (never) & 47.4 & 19.7 & $52.68,1,0.001$ \\
\hline
\end{tabular}

age, gender and educational status. There were no significant differences between the two groups in terms of marital status or social classification.

These data, however, indicate that the CFS group were significantly less likely to be in employment than the controls at the time of testing.

Although, as stated above, the two groups were matched in terms of educational status, the CFS group made significantly more errors on the pre-morbid intelligence test than the controls (see Table 1).

\section{Health-Related Behaviours}

Although there was no significant difference between the average number of hours slept by the two groups, the CFS group was significantly less likely to feel rested from sleep (see Table 1). The CFS group were significantly more likely to be taking prescribed medication and dietary supplements than the control group. The controls were also significantly more likely to smoke and consume alcohol than the patient group and were more likely to exercise regularly. There were no differences between the two groups in terms of food intake.

\section{Symptoms and Illness Severity}

When considering the symptom check-list, there were statistically significant differences between the CFS and control groups on each of the 28 items on the scale $(p<0.001)$ and, therefore, the mean total symptom scores for the CFS patients was significantly higher than the control group. As one might expect in this group of patients, the CFS group recorded higher fatigue ratings on the Profile of Fatigue Related Symptoms scale [28]. On this scale they also recorded significantly higher levels of emotional distress, cognitive difficulties and somatic symptoms (see Table 2 ).

\section{Psychopathology, Mood and Cognition}

Participants were asked to rate their responses relative to the previous week including the day of testing (see Table $\mathbf{3}$ ).

Table 2. Symptom and Illness Severity Scores (from the PFRS Scale) for the CFS and Control Sample. Scores are the Group Means with s.e.m in Parenthesis. Higher Scores=Greater Symptom/Illness Severity

\begin{tabular}{|c|c|c|c|}
\hline & CFS $(\mathbf{N}=\mathbf{3 0 7})$ & Controls $(\mathbf{N}=126)$ & F, df, p \\
\hline \hline Symptoms: & & & \\
\hline Total Symptoms $(\mathrm{n}=28)$ & $15.76(0.32)$ & $2.69(0.28)$ & $618.90,1426,0.001$ \\
\hline Fatigue Related Symptoms: & & & $53.56,1426,0.001$ \\
ED & $47.80(1.21)$ & $32.51(1.40)$ & $679.10,1424,0.001$ \\
Fatigue & $62.90(0.89)$ & $23.05(1.04)$ & $226.50,1426,0.001$ \\
CD & $46.89(0.92)$ & $23.58(0.94)$ & $269.20,1424,0.001$ \\
SS & $53.27(1.13)$ & $23.51(0.78)$ & \\
\hline
\end{tabular}

$\mathrm{ED}=$ Emotional Distress; $\mathrm{CD}=$ Cognitive Difficulties; $\mathrm{SS}=$ Somatic Symptoms . 
Table 3. Subjective Measures of Psychopathology, Mood and Cognition for the CFS and Control Samples. Scores are the Group Means with s.e.m in Parenthesis

\begin{tabular}{|c|c|c|c|}
\hline & $\begin{array}{c}\text { CFS } \\
(\mathbf{N}=307)\end{array}$ & $\begin{array}{l}\text { Controls } \\
(\mathrm{N}=126)\end{array}$ & $\mathbf{F}, \mathbf{d f}, \mathbf{p}$ \\
\hline \multicolumn{4}{|l|}{ Psychopathology, Mood and Cognition: } \\
\hline Depression $(\uparrow$ scores $=\uparrow$ dep. $)$ & $\begin{array}{l}41.46 \\
(0.61)\end{array}$ & $\begin{array}{l}35.07 \\
(0.48)\end{array}$ & $41.58,1424,0.001$ \\
\hline Trait Anxiety ( $\uparrow$ scores $=\uparrow$ anx.) & $\begin{array}{l}49.36 \\
(0.66)\end{array}$ & $\begin{array}{l}40.45 \\
(0.97)\end{array}$ & $55.50,1421,0.001$ \\
\hline $\begin{array}{c}\text { Mood: } \\
\text { Alertness } \\
(\uparrow \text { scores }=\uparrow \text { alert. }) \\
\text { Hedonic tone } \\
(\uparrow \text { scores }=\uparrow \mathrm{HT}) \\
\text { Anxiety } \\
(\downarrow \text { scores }=\uparrow \text { anx. })\end{array}$ & $\begin{array}{c}188.73 \\
(1.35) \\
122.78 \\
(1.15) \\
74.15 \\
(1.04)\end{array}$ & $\begin{array}{c}290.27 \\
(6.00) \\
234.17 \\
(4.10) \\
105.29 \\
(2.67)\end{array}$ & $\begin{array}{c}536.70,1431,0.001 \\
1227.05,1431,0.001 \\
174.23,1431,0.001\end{array}$ \\
\hline $\begin{array}{c}\text { Positive Mood } \\
(\uparrow \text { scores }=\uparrow \text { pos. } \mathrm{md}) \\
\text { Negative Mood }(\uparrow \text { scores }=\uparrow \text { neg. } \mathrm{md})\end{array}$ & $\begin{array}{l}25.88 \\
(0.56) \\
23.95 \\
(0.66)\end{array}$ & $\begin{array}{l}36.05 \\
(0.82) \\
14.14 \\
(0.85)\end{array}$ & $\begin{array}{l}98.16,1424,0.001 \\
71.44,1425,0.001\end{array}$ \\
\hline Cognitive Failures $(\uparrow$ scores $=\uparrow \mathrm{CF})$ & $\begin{array}{l}60.53 \\
(1.03)\end{array}$ & $38.35(1.17)$ & $156.00,1419,0.001$ \\
\hline
\end{tabular}

These data suggest that the CFS patients were more depressed than the controls. In response to the more generalised questions posed in the pre-visit booklet, the trait anxiety scores for the CFS patients were significantly higher than those of the controls as were the levels of cognitive failures. However, there was no significant difference between the two groups in terms of anxiety $(\mathrm{CFS}=40.42$, controls $=41.67$; $\mathrm{p}=0.20)$ when the participant was asked to rate how the felt on the day of testing. The CFS group did report lower positive and higher negative mood scores than the controls during the previous week. These mood data also showed that the CFS group were significantly less alert than the controls, had lower hedonic tone scores and were more anxious than the control group (see Table $\mathbf{3}$ ).

\section{Psychosocial Measures}

The CFS group reported significantly higher levels of perceived stress, suffered more severe daily hassles and less positive life events than the controls. However, there were no significant differences between the groups in terms of self-esteem or negative life events (see Table 4).

\section{Performance Testing}

Data comparing the CFS and controls in terms of performance measures are depicted graphically in Fig. (1).

\section{Free Recall}

The CFS patients recalled significantly fewer words than the healthy controls indicating deficits in episodic memory in the patient group $(\mathrm{CFS}=6.12$ words, controls=7.48 words; $\mathrm{F}(1,430)=39.92, \mathrm{p}<0.001)$.

\section{Simple Reaction Time Task}

The mean reaction time (mRT) over the 3 minutes of the test was significantly longer for the CFS patients than the controls $(\mathrm{CFS}=490.81 \mathrm{msec}$, controls $=284.15 \mathrm{msec} ; \mathrm{F}(1$, 430) $=34.30, \mathrm{p}<0.001)$.

Table 4. Psychosocial Measures for the CFS and Control Samples. Scores are the Group Means with s.e.m in Parenthesis

\begin{tabular}{|c|c|c|c|}
\hline & CFS $(\mathrm{N}=307)$ & Controls $(\mathrm{N}=126)$ & $F$, df, $p$ \\
\hline Daily Hassles $(\uparrow$ scores $=\uparrow$ severity) & $46.51(2.06)$ & $32.73(2.76)$ & $14.26,1421,0.001$ \\
\hline $\begin{array}{c}\text { Self Esteem } \\
(\uparrow \text { scores }=\uparrow \mathrm{SE})\end{array}$ & $57.32(0.92)$ & $58.91(1.30)$ & $\mathrm{n} / \mathrm{s}$ \\
\hline $\begin{array}{l}\text { Perceived Stress } \\
(\uparrow \text { scores }=\uparrow \text { stress })\end{array}$ & $26.76(0.48)$ & $22.55(0.77)$ & $21.851,1421,0.001$ \\
\hline $\begin{array}{l}\text { Positive Life Events }(\uparrow \text { scores }=\uparrow \mathrm{PLE}) \\
\text { Negative Life Events }(\uparrow \text { scores }=\uparrow \mathrm{NLE})\end{array}$ & $\begin{array}{l}0.78(0.07) \\
2.34(0.13)\end{array}$ & $\begin{array}{l}1.26(0.13) \\
2.66(0.21)\end{array}$ & $\begin{array}{c}12.65,1426,0.001 \\
\mathrm{n} / \mathrm{s}\end{array}$ \\
\hline
\end{tabular}


a) Episodic memory

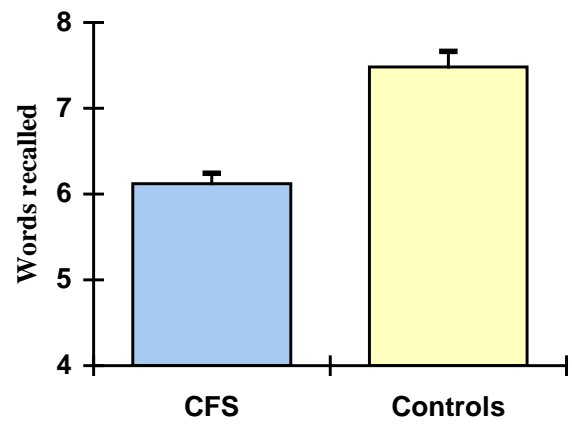

b)

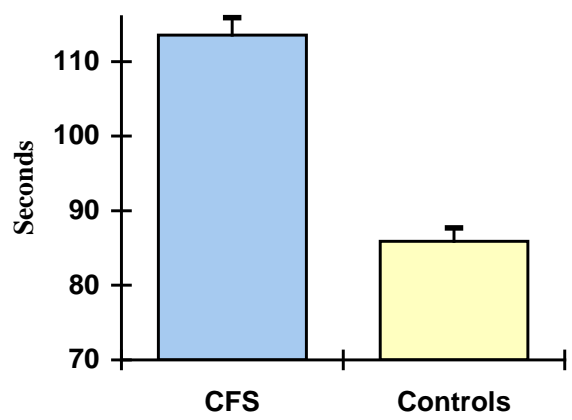

c)

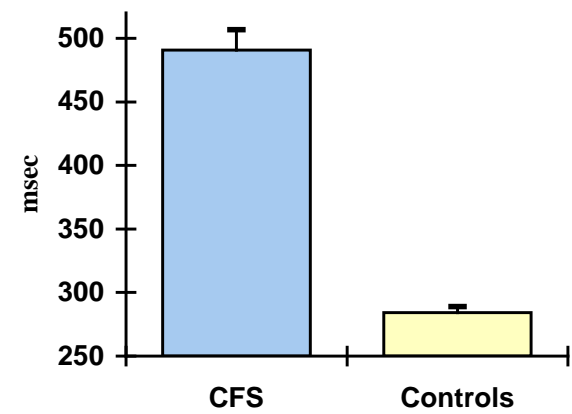

d)

Vigilance

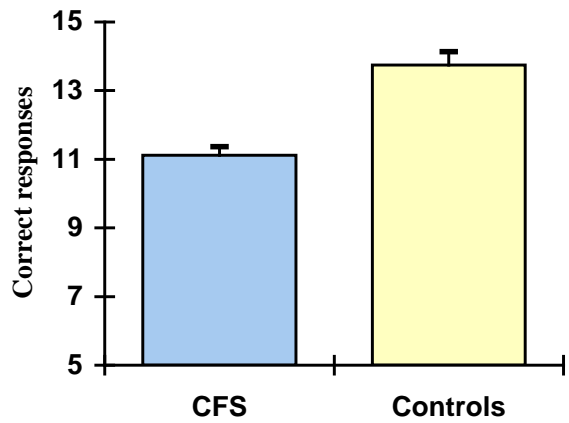

Fig. (1). Objective measures of performance for the CFS and control groups. Scores are the mean with s.e.m shown as bars. Higher Stroop and RT scores=slower reaction times.

Data describing the time-on-task reaction times over the 3 minutes (that is the RT for each minute of the task) suggests that there is a linear group effect for reaction times $(F(1,429)=67.20, p<0.001)$ indicating that both groups' reac- tion times slow gradually over the three minutes. CFS patients had slower reaction times on each minute of the task (time on task), and the repeated measures analysis indicated that the CFS groups' reaction time slowed at a greater rate $(\mathrm{F}(1,858)=3.03, \mathrm{p}<0.05)$ than the controls (i.e. they become fatigued more quickly than the controls - see Fig. 2).

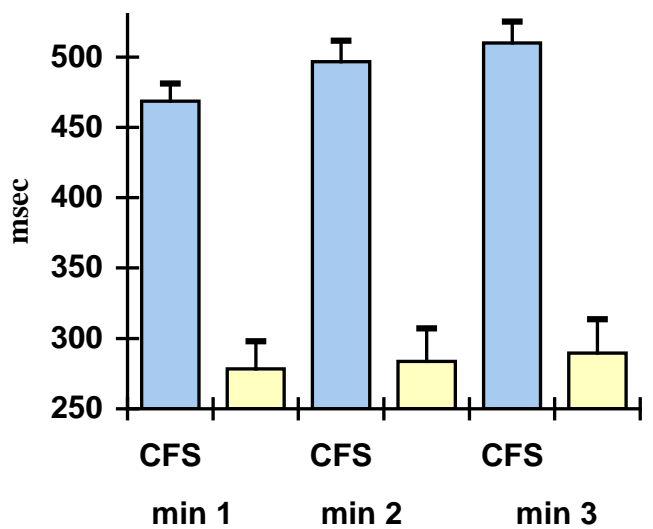

Fig. (2). Time on task scores for three minutes of the simple reaction time task measure for the CFS and control groups. Scores are the mean with s.e.m shown as bars. Higher RT scores=slower reaction times.

\section{Repeated Digits Vigilance Task}

Again the overall mean reaction time for the CFS patients on this task was significantly slower than the controls $(\mathrm{CFS}=611.7 \mathrm{msec}, \quad$ controls $=549.4 \mathrm{msec} ; \quad \mathrm{F}(1,383)=26.80$, $\mathrm{p}<0.001)$. There was no time-on-task effect in the vigilance task even though group differences in the reaction times for each minute on the task remain. The total number of correctly detected targets (hits) was also significantly lower in the CFS group $(\mathrm{CFS}=11.12$ hits, control=13.75 hits; $\mathrm{F}(1$, $423)=34.30, \mathrm{p}<0.001)$ but there no overall time-on-task group effect. The control group detected significantly fewer hits in minute 2 when compared to minute 1 ( $\min 1=5.31$, $\min 2=4.94 ; \mathrm{F}(2,124)=59.33, \mathrm{p}<0.01)$ and significantly fewer hits in minute 3 compared to minute 2 ( $\min 2=4.94$, $\min 3=3.50 ; \mathrm{F}(2,124)=59.33, \mathrm{p}<0.001)$. However, although the CFS group detected significantly fewer hits in minute 3 than minute $2(\min 2=4.24, \min 3=2.81 ; \mathrm{F}(2,296)=86.21$, $\mathrm{p}<0.001)$ there was no difference between the number of hits detected between minutes 1 and 2 .

There was no difference between the two groups in terms of false alarms.

\section{Distraction from Irrelevant Stimuli}

Analysis of covariance on the Stroop test, with the word control condition as a covariate, produced no significant differences between the groups when measuring colour interference (name the word, ignore the colour). The CFS group, however were significantly slower than the controls when naming the colour and ignoring the word (CFS=113.52 seconds, controls $=85.91$ seconds; $F(1,427)=52.68, \mathrm{p}<0.001)$.

\section{IMPAIRED COGNITION AND POSSIBLE CON- FOUNDING FACTORS}

As factors such as demographics, symptoms, anxiety, depression, mood and psychosocial factors have been shown 
Table 5. Confounding Factors which are Known to Exert an Independent Effect on Cognition. Factors Ultimately Identified in the Analyses are Highlighted in Bold

\begin{tabular}{|c|c|c|c|}
\hline $\begin{array}{c}\text { Stroop } \\
\text { Word Interference }\end{array}$ & $\begin{array}{c}\text { Free Recall } \\
\text { Words Recalled }\end{array}$ & $\begin{array}{c}\text { Simple Reaction Time } \\
\text { Mean Reaction Time }\end{array}$ & $\begin{array}{l}\text { Repeated Digits Task } \\
\text { Digits Detected }\end{array}$ \\
\hline $\begin{array}{c}\text { Gender } \\
\text { Age } \\
\text { Educational Status } \\
\text { Employment Status } \\
\text { NART }\end{array}$ & $\begin{array}{c}\text { Age } \\
\text { Marital Status } \\
\text { Educational Status } \\
\text { Employment Status } \\
\text { Social Classification } \\
\text { NART }\end{array}$ & $\begin{array}{c}\text { Gender } \\
\text { Marital Status } \\
\text { Educational Status } \\
\text { Employment Status } \\
\text { Social Classification } \\
\text { NART }\end{array}$ & $\begin{array}{c}\text { Gender } \\
\text { Educational Status } \\
\text { Employment Status } \\
\text { Social Classification } \\
\text { NART }\end{array}$ \\
\hline Rested by sleep & Rested by sleep & $\begin{array}{c}\text { Medication } \\
\text { Rested by sleep }\end{array}$ & Rested by sleep \\
\hline $\begin{array}{l}\text { Emotional Distress } \\
\text { Cognitive Difficulties } \\
\text { Somatic Symptoms } \\
\text { Physical Symptoms }\end{array}$ & $\begin{array}{c}\text { Emotional Distress } \\
\text { Cognitive Difficulties } \\
\text { Somatic Symptoms } \\
\text { Physical Symptoms }\end{array}$ & $\begin{array}{l}\text { Emotional Distress } \\
\text { Cognitive Difficulties } \\
\text { Somatic Symptoms } \\
\text { Physical Symptoms }\end{array}$ & $\begin{array}{c}\text { Emotional Distress } \\
\text { Cognitive Difficulties } \\
\text { Somatic Symptoms }\end{array}$ \\
\hline $\begin{array}{c}\text { Depression } \\
\text { Trait Anxiety } \\
\text { Positive Mood } \\
\text { Negative Mood } \\
\text { Cognitive Failures }\end{array}$ & $\begin{array}{c}\text { Depression } \\
\text { Trait Anxiety } \\
\text { Positive Mood } \\
\text { Negative Mood } \\
\text { Cognitive Failures }\end{array}$ & $\begin{array}{c}\text { Depression } \\
\text { Trait Anxiety } \\
\text { Positive Mood } \\
\text { Negative Mood } \\
\text { Cognitive Failures }\end{array}$ & $\begin{array}{c}\text { Depression } \\
\text { Positive Mood } \\
\text { Cognitive Failures }\end{array}$ \\
\hline $\begin{array}{c}\text { Daily Hassles } \\
\text { Perceived Stress }\end{array}$ & $\begin{array}{c}\text { Daily Hassles } \\
\text { Perceived Stress }\end{array}$ & $\begin{array}{c}\text { Daily Hassles } \\
\text { Perceived Stress }\end{array}$ & Daily Hassles \\
\hline
\end{tabular}

to be associated with impaired cognition in previous studies, a series of univariate analyses were conducted on each performance task using the individual variables listed in Tables 1-3 as covariates. From these analyses only two variables produced an independent effect which removed the overall group difference in cognition between the CFS and controls; total symptoms and fatigue scores. Table $\mathbf{5}$ describes the factors exerting an independent effect for each performance task whilst not affecting the differences between the CFS and control groups.

When all the independent factors were co-varied in one analysis, several measures had a significant independent effect (shown in bold) but again the difference between CFS patients and controls only became non-significant when fatigue and symptom severity were co-varied.

\section{SUBJECTIVE MEASURES OF COGNITION VERSUS OBJECTIVE MEASURES OF PERFORMANCE}

The Cognitive Failures Questionnaire (CFQ) was used in this study as a subjective measure of cognitive impairment. To investigate the suggestion that patients with CFS overestimate the levels of cognitive deficits experienced, a median split was performed on the CFQ scores for each of the two groups and, as a result, four sub-groups were created: (a) CFS patients with low CFQ scores $(n=107)$, (b) controls with low CFQ scores $(n=106)$, (c) CFS patients with high CFQ scores $(n=186)$ and, (d) controls with high CFQ scores $(n=16)$. These were then used as grouping factors in analyses of variance for each of the performance measures with the independent factors highlighted in Table $\mathbf{5}$ as covariates. These results are shown in Table $\mathbf{6}$.

Table 6. Subjective Measures of Cognition (Cognitive Failures) Versus Objective Measures of Performance. Scores are the Group Means with s.e.m in Parenthesis. Higher Stroop and RT Scores=Slower Reaction Times

\begin{tabular}{|c|c|c|c|c|}
\hline & $\begin{array}{c}\text { Stroop } \\
(\text { sec. })\end{array}$ & Free Recall & $\begin{array}{c}\text { Simple Reaction Time } \\
(\text { msec.) }\end{array}$ & $\begin{array}{c}\text { Repeated Digits Task } \\
\text { (Detected) }\end{array}$ \\
\hline \hline Low CFQ: & & & $400.70(21.73)$ & $11.77(0.40)$ \\
CFS & $101.69(3.14)$ & $6.26(0.20)$ & $320.27(22.26)$ & $12.68(0.43)$ \\
Controls & $88.48(3.16)$ & $6.79(0.23)$ & $523.25(16.91)$ & $11.33(0.32)$ \\
High $\boldsymbol{C F Q}:$ & $117.95(2.40)$ & $6.44(0.19)$ & $282.68(55.02)$ & $14.64(1.00)$ \\
CFS & $89.14(8.12)$ & $7.41(0.45)$ & & \\
Controls & $\mathrm{F}(3,409)=19.94, \mathrm{p}<0.001$ & $\mathrm{~F}(3,409)=2.93, \mathrm{p}<0.033$ & $\mathrm{~F}(3,406)=19.398, \mathrm{p}<0.001$ & $\mathrm{~F}(3,403)=4.15, \mathrm{p}<0.006$ \\
\hline Group Effect: & & &
\end{tabular}


There is an overall group effect of CFQ on cognition and, therefore, an association between the subjective CFQ measure and level of cognitive deficit in CFS.

To further investigate the relationship between subjective and objective measures, the cognitive failures questionnaire scores for the patient and control groups were split into their respective quartiles and used as grouping factors.

These analyses reveal clear differences between the CFQ quartiles for the CFS group for both the mean reaction time task $(\mathrm{F}(3,285)=3.32, \mathrm{p}<0.02)$ and the Stroop task $(\mathrm{F}(3$, $287)=4.78, \mathrm{p}<0.003$ ), but not for the free recall or vigilance tasks. For the controls, however, there were no associations between CFQ scores and deficits for any of the performance measures.

\section{Illness Severity and Performance}

Two measures, namely the total symptom scores and the Profile of Fatigue Related Symptoms (PRFS), were used to assess illness severity in the patient sample. To investigate the relationship between illness severity and cognitive deficits, these two measures were dichotomised and summed to create six groups (a) low illness severity CFS patients $(\mathrm{n}=61)$, (b) low illness severity controls $(\mathrm{n}=117)$, (c) medium illness severity CFS ( $\mathrm{n}=57$ ), (d) medium illness severity controls $(n=8)$, (e) high illness severity CFS $(n=175)$ and, (f) high illness severity controls. Unfortunately, there were no controls in the high illness severity grouping and only eight in the medium severity control group. Data for the medium illness severity controls have been included in Table 7 (which describes these data for each performance task) for comparison purposes but will not be discussed further.

There was an overall group effect for free recall scores, with there being significant differences between the low illness severity controls and the medium and high illness severity patients $(p<0.001$ and $p<0.002$ respectively). However, there were no indications of an association between illness severity and performance deficits in the CFS groups.

There was also an overall group effect in the simple reaction time task, with there being significant differences between the low illness severity controls and the low, medium and high illness severity patients $(\mathrm{p}<0.04, \mathrm{p}<0.001$ and $\mathrm{p}<0.001$ respectively). In addition, there were significant differences in the simple reaction times between the low and high illness severity patients $(p<0.001)$ and a marginal dif- ference between low and medium illness severity patients $(\mathrm{p}=0.06)$. However, there was no significant difference between the medium and high illness severity patients.

There was an overall group effect in the vigilance task, with significant differences between the low illness severity controls and the medium and high illness severity patients $(\mathrm{p}<0.01$ and $\mathrm{p}<0.001$ respectively). However, in this task there were no indications of an association between illness severity and performance deficits in the CFS groups.

There was an overall group effect in word interference, with there being significant differences between the low illness severity controls and the low, medium and high illness severity patients $(\mathrm{p}<0.05, \mathrm{p}<0.03$ and $\mathrm{p}<0.001$ respectively). In addition, there were significant differences in the time taken to complete the task by the low and high illness severity patients $(p<0.001)$ and the medium and high illness severity patients $(\mathrm{p}<0.001)$. However, there was no significant difference between the low and medium illness severity patients.

These data provide a similar profile to that of cognitive failures: that is, there were associations between the level of illness severity and cognition for the two reaction time tasks but not for the recall and vigilance tasks.

\section{DISCUSSION}

The current study set out to bring some clarity to the issues arising from the on-going debate surrounding the exact nature, and indeed existence, of the cognitive deficits associated Chronic Fatigue Syndrome (CFS). This was achieved by addressing specific comments made by previous reviewers of the literature $[17,18]$ as well incorporating into the design the views of those who found no evidence of such impairment $[7,15,16]$.

To begin with we recruited 307 participants, a much larger number of patients than previous studies, from a single specialised outpatient clinic. To address the issue of accuracy of diagnosis, the Centre for Disease Control (CDC) case definition criteria [2] was used to diagnose all patients invited to take part in the study, which excludes patients exhibiting major psychiatric disorders. It was clear that our sample conformed to the expected demographic profile for Chronic Fatigue Syndrome (CFS): that is, a predominance of middleaged married females.

Table 7. Illness Severity Versus Cognitive Performance. Scores are the Group Means with s.e.m in Parenthesis. Higher Stroop and RT Scores=Slower Reaction Times

\begin{tabular}{|c|c|c|c|c|}
\hline & $\begin{array}{c}\text { Stroop } \\
(\mathrm{sec})\end{array}$ & $\begin{array}{c}\text { Free Recall } \\
(\text { words })\end{array}$ & $\begin{array}{c}\text { Simple Reaction Time } \\
(\mathrm{msec})\end{array}$ & $\begin{array}{c}\text { Repeated Digits Task } \\
(\mathrm{digits})\end{array}$ \\
\hline Low Illness Severity: & & & $387.23(29.07)$ & $11.98(0.51)$ \\
CFS & $98.32(4.04)$ & $6.70(0.24)$ & $315.00(21.35)$ & $13.11(0.41)$ \\
Controls & $88.31(2.94)$ & $7.04(0.20)$ & $466.73(29.33)$ & $11.38(0.54)$ \\
Medium Illness Severity: & $99.185(4.18)$ & $6.23(0.24)$ & $325.41(78.21)$ & $12.52(1.41)$ \\
CFS & $92.07(11.25)$ & $6.87(0.63)$ & $516.30(17.72)$ & $11.21(0.33)$ \\
Controls & $120.62(2.40)$ & $6.21(0.16)$ & $\mathrm{F}(4,404)=13.32, \mathrm{p}<0.000$ & $\mathrm{~F}(4,405)=2.94, \mathrm{p}<0.020$ \\
High Illness Severity: & $\mathrm{F}(4,411)=19.78, \mathrm{p}<0.000$ & $\mathrm{~F}(4,404)=2.64, \mathrm{p}<0.034$ & \\
CFS & & & & \\
\hline Group Effect: & &
\end{tabular}


When examining the patient sample in more detail we found that the majority of the group (68\%) had received a preliminary diagnosis of CFS from their GP before attending the clinic and their mean illness length was five years. Although the majority of patients suggested a physical cause as a possible trigger for their illness (such as influenza), several patients acknowledged a non-physical cause (for example, a stress-related episode).

Half the sample of patients experienced fluctuations in symptom severity although no regular daily pattern emerged. The resultant effect of such variability is that patients experience high levels of uncertainty. Data from a symptom check list provided evidence of the myriad symptoms experienced by CFS sufferers. The study showed that and the majority of patients $(41 \%)$ reported their condition as 'bad with some recovery' on the 5-item current state of health scale at the time of testing. Increased rest and sleep were highlighted by patients as the most effective approaches in their attempt to minimise the negative effects of the illness whilst, on the other hand, exercise, walking, shopping, mental effort and stress were most commonly linked with an exacerbation of symptom severity.

To address criticisms aimed at the inclusion of inappropriate control groups in previously reported studies, we recruited 126 healthy volunteers from a cross-section of the general population who were matched to the patient sample in terms of age, gender, educational standard and socioeconomic group. In addition, the controls completed a similar screening process to the patient group.

Concerns voiced by Wearden and Appleby [18] regarding the suitability and sensitivity of the objective measures of cognitive performance used in previous research were answered by administering tasks which, not only reflected the type of cognitive impairments patients themselves report, but the tests used had been utilised in previous studies of the illness $[8,25]$ and in studies of fatigue in healthy individuals [6].

In a series of group comparisons we found several differences between the CFS patients and control group. Firstly, and not surprisingly considering the severity of the illness, the controls were significantly more likely to be in employment than the CFS group at the time of testing. Secondly, despite the two groups being matched in terms of educational status, the CFS patients scored significantly lower on the pre-morbid intelligence measure than the controls. There are two possible explanations for this: (a) as intelligence shows a positive correlation with most cognitive measures [39], the converse is also true, namely that deficits in cognitive functioning will be accompanied by lower intelligence scores; (b) patients with depression have been shown to report lower pre-morbid intelligence scores using the NART score [40].

A further area of interest is the role of sleep abnormalities in this illness [8]. Our data suggests that the average number of hours slept per night did not differ between the patient and control groups. However, the two groups were shown to differ in ratings of the extent to which the individual felt rested as a result of sleep - namely sleep quality. Abnormalities in sleep patterns had been shown to affect mood and performance both in CFS patients and healthy controls $[8,27]$ and were an important factor to consider when examining these data further. In addition, problems with sleep quality are often observed in abnormal psychopathology [41, 42].

The presence of co-morbid mood disorder is also indicated in the patient group. Although these findings contradict previous findings (e.g. Marshall et al. 1996 [14]), it is believed that this study provides a more accurate representation of the illness due to the fact that a larger cohort was assessed in comparison with previous studies. Indeed laboratory testing further highlight the differences between the mood states of the two groups. The CFS patients record significantly lower levels alertness and hedonic tone and have higher anxiety levels than the control group. It is of note that the state anxiety scores of the two participant groups do not differ at the time of testing, indicating that the patient group was not unduly distressed by the test session itself. Trait anxiety scores, however, indicate that patient group exhibit higher general anxiety levels than the controls.

Simple comparisons of performance task data seem to confirm our previous findings of a slowing of motor speed and impairments in immediate recall in CFS. The reaction time task also provides data indicating that there were significant differences between the reaction times of the CFS and control groups when comparing reaction times at minute intervals. In addition, a greater rate of slowing over the three minutes was seen in the CFS group. This 'time-on-task effect' was not replicated in the other three minute task (the vigilance task) even though group differences in the reaction times for each minute on the task remain. There is a suggestion here that although both sustained attention and motor speed are affected in CFS, time-on-task effects differ. A possible explanation may be that performance on such tasks are affected differently by fatigue and that these differences may reflect differences in underlying neurotransmitter mechanisms involved in processing the two tasks (Christopher et al. 2005 for a discussion [43]). However, this theory would have to be tested in further wide-ranging studies.

Returning to the vigilance task, the detection rate of repeated digits for each minute was greater in the control group than the patient group and both groups' detection rate decreased over time. In contrast to our previous findings, where a gradual decline in accuracy over time was reported for both groups [25], the current study indicates that there was no measurable decline in accuracy for the patient group between minutes 1 and 2. A trade-off between reaction time and accuracy (for minutes 1 and 2) is, however, observed in healthy controls and this warrants further investigation.

The final performance task, the Stroop interference task [37], provided evidence to suggest that distraction by irrelevant stimuli is more pronounced in the patient group. These data are contrary to findings reported by Metzger and Denny [44] who found no evidence to suggest that CFS patients perform differently to controls on this task. The inconsistency between their study and the results presented here might be due to the relatively small number of patients tested (40 compare to 307) and the fact that a modified version of the Stroop task was used to assess cognitive function. By modifying the task, some test sensitivity may have been lost and this has been suggested as a possible reason for the lack of conclusive data in the past. 
To investigate these data further, a series of additional analyses were conducted, this time identifying a range of factors significantly associated with poorer performance and including these as covariates in the analyses. These included demographic data, pre-morbid intelligence, illness severity, anxiety, depression, psychosocial factors and sleep data. Only two variables, total symptoms and the fatigue sub-scale of the fatigue-related symptoms questionnaire, were identified as factors which act to remove the overall group effect on performance. Although one might expect the level of somatic symptoms, cognitive difficulties and emotional distress to be major factors of influence in this particular illness, these two factors essentially define the two groups. However, several factors were identified as having an independent effect on performance without removing the overall group effect. These factors were, therefore, used as covariates in further analyses to ensure that they could act in cumulative way and affect performance. There was no evidence to suggest that co-morbid anxiety and/or depression play a role in the level of cognitive deficit in this illness.

In order to consider the ability of the patient group to subjectively assess their level of cognitive impairment accurately, data from the Cognitive Failures Questionnaire (CFQ) were used [32]. Previous studies showed that the subjective ratings of cognitive deficits by CFS patients were much higher than the level of deficits identified on objective measures of performance. Patients recording high scores on the Cognitive Failures Questionnaire performed significantly worse than those recording low scores for the two reaction time tasks. These data indicate that patients are more able to accurately subjectively rate their cognitive performance than previous reports have indicated [18].

It was also important to investigate whether illness severity negatively affects cognitive functioning, that is are the more severe cases of CFS more functionally impaired. Illness severity as weighted by splitting the patients according to their fatigue-related symptoms and total symptom scores. These scores were then summed and compared to each performance task allowing us to look at performance across a range of severity scores. The controls performed better on each of the tasks than the patient groups even those rated with low illness severity. When considering the CFS group alone, an association between illness severity and poorer cognitive performance is seen in the psychomotor tasks but not the accuracy or recall tasks. These outcomes are partly in agreement with previous studies $[17,18]$ but also raises further questions as to the exact nature of the cognitive impairment associated with CFS.

Recruiting patients via a specialised out-patients raises issues about generalisability and this is acknowledged as a limitation of the study. However, our data indicating a measurable deficit in motor speed are in keeping with findings from a recent population-based study [45].

\section{CONCLUSION}

The current study provides compelling evidence, not only for the existence of cognitive deficits in Chronic Fatigue Syndrome, but for the nature of these same impairments. Of interest here is that these objectively measured deficits remain even when factors associated with performance impairments are taken into consideration. Improvements or recovery from the illness should, therefore, be accompanied by improvements in cognition. The possibility of measuring recovery in these terms is indicated here: indeed, recent research provides evidence that some of the performance measures used in this study may be valid indicators of recovery [46]. Further studies are, however, required to investigate the possible mechanisms underlying our findings. One area of interest may be the influence of specific neurotransmitters such as acetylcholine, noradrenalin and dopamine in cognitive functioning. Another is the role of insulin in modulating acetylcholine and noradrenalin which in turn influences cognitive functioning. Similarly, dopamine has also been shown to influence motor functioning, verbal fluency, episodic memory and executive functioning [47, 48]. In addition, several groups have identified neuro-anatomical abnormalities in patients with CFS using neuroimaging techniques. These include reduced regional cerebral blood flow, anatomical abnormalities in cortical and subcortical regions and reduced glucose metabolism. The impact of such abnormalities are likely to impact on cognitive performance and may go some way in offering an explanation for some of the cognitive impairments observed in the current study.

There remains a large gap in the current body of scientific knowledge as to the nature of these abnormalities. One area of interest is 'cause and effect': that is, do the abnormalities observed cause CFS or does the decrease in physical activity resulting from the illness produce the abnormality. For example, there have been reports of a link between physical activity and neurogenesis [49]. In addition, recent studies have also suggested that neurogenesis is involved in the action of antidepressants [50]. These findings may explain the increased recovery rates detected in CFS patients prescribed antidepressant medication in our study [51].

Finally, there is an emerging hypothesis based on the model of 'altered self' which refers to the immune response to infection [52]. Infectious diseases are often accompanied or followed by periods of fatigue, disturbed sleep and an inability to concentrate. The theory is widened to a brain function model where an extended concept of altered self means that the patient does not return to the non-disease state due to the brains failure to recognise that state [52].

\section{ACKNOWLEDGEMENTS}

This study was funded by the Linbury Trust and the Gatsby Foundation.

\section{REFERENCES}

[1] Sharpe M, Archard L, Banatvala J, et al. CFS: guidelines for research. J R Soc Med 1991; 84: 118-21.

[2] Fukuda K, Straus S, Hickie I, Sharpe MC, Dobbins JG, Komaroff A; International Chronic Fatigue Syndrome Study Group. The Chronic Fatigue Syndrome: a comprehensive approach to its definition and study. Ann Intern Med 1994; 121: 953-9.

[3] Surawy C, Hackman A, Hawton K, Sharpe M. CFS: A cognitive approach. Behav Res Ther 1995; 33: 535-44.

[4] van der Linden D, Frese M, Meijman T. Mental fatigue and the control of cognitive process: effects on preservation and planning. Acta Psychol 2003; 113: 45-65.

[5] Boksem MAS, Meijman TF, Lorist MM. Effects of mental fatigue on attention: and ERP study. Brain Res Cogn Brain Res 2005; 25: 107-16.

[6] Smith AP, Brockman P, Flynn R, Maben A, Thomas M. An investigation of the effects of coffee on alertness and performance during the day and night. Neuropsychobiology 1993; 27: 217-33. 
[7] LaManca JJ, Sisto SA, DeLuca J, et al. Influence of exhaustive treadmill exercise on cognitive functioning in chronic fatigue syndrome. Am J Med 1998; 105: 59S-65S

[8] Smith A, Pollock J, Thomas M, Llewelyn M, Borysiewicz L. The relationship between subjective ratings of sleep and mental functioning in healthy subjects and patients with chronic fatigue syndrome. Hum Psychopharmacology 1996; 11: 161-7.

[9] Lawrie SM, MacHale, Cavanagh JT, O'Carroll RE, Goodwin GM. The difference in patterns of motor and cognitive function in chronic fatigue syndrome and severe depression. Psychol Med 2000; 30: 433-42.

[10] Davey NJ, Puri BK, Nowicky AV, Main J, Zaman R. Voluntary motor function in patients with chronic fatigue syndrome. J Psychosomatic Res 2001; 50: 17-20.

[11] Capuron L, Welberg L, Heim C, et al. Cognitive dysfunction relates to subjective report of mental fatigue in patients with chronic fatigue syndrome. Neuropsychopharmacology 2006; 31: 1777-84.

[12] Claypole KH, Noonan C, Mahurin RK, Goldberg J, Erickson T, Buchwald D. A twin study of cognitive function in chronic fatigue syndrome: The effects of sudden illness onset. Neuropsychology 2007; $21: 507-13$.

[13] Marcel B, Komaroff AJ, Faioli LR, Kornish RJ, Albert MS. Cognitive deficits in patients with CFS. Biol Psychiatry 1996; 40: 53541.

[14] Marshall PS, Watson D, Steinberg P, et al. An assessment of cognitive function and mood in CFS. Biol Psychiatry 1996; 39: 199-206.

[15] Short K, McCabe M, Tooley G. Cognitive functioning in CFS and the role of depression, anxiety and fatigue. J Psychosomatic Res 2002; 52: 475-83.

[16] DeLuca J, Christodoulou C, Diamond BJ, et al. The nature of memory impairment in chronic fatigue syndrome. Rehab Psychol 2004; 49: 62-70.

[17] Fry AM, Martin M. Fatigue in the Chronic Fatigue Syndrome: A cognitive phenomenon? J Psychosomatic Res 1996; 41: 415-26.

[18] Wearden AJ, Appleby L. Research on cognitive complaints and cognitive functioning in patients with chronic fatigue syndrome: What conclusions can we draw? J Psychosomatic Res 1996; 41: 197-211.

[19] Jensen AR. Individual differences in the Hick paradigm. Speed and information processing and intelligence. Norwood, New Jersey: Ablex Publishing 1987; pp. 101-75.

[20] Brown RG, Scott LC, Bench CJ, Dolan RJ. Cognitive function in depression: its relationship to the presence and severity of intellectual decline. Psychol Med 1994; 24: 829-47.

[21] Christopher G, MacDonald J. The impact of clinical depression on working memory. Cognit Neuropsychiatry 2005; 10: 379-99.

[22] Eysenck MW, Calvo MG. Anxiety and performance: the processing efficiency theory. Cogn Emot 1992; 6: 409-34.

[23] Michiels V, Cluydts R. Neuropsychological functioning in Chronic Fatigue Syndrome: a review. Acta Psychiatr Scand 2001; 103: 84-93.

[24] Wessely S, Chalder T, Hirsch S, Wallace P, Wright D. Psychological symptoms, somatic symptoms and psychiatric disorder in chronic fatigue and CFS: a prospective study in primary care. Am J Psychiatry 1996; 153: 1050-9.

[25] Smith AP, Borysiewicz L, Pollock J, Thomas M, Perry K, Llewelyn M. Acute fatigue in chronic fatigue syndrome. Psychol Med 1999; 29: 283-90

[26] Nelson HE. National Adult Reading Test. Windsor, UK: NFERNelson 1982.

[27] Smith AP, Behan PO, Bell W, Millar K, Bakheit M. Behavioural problems associated with the chronic fatigue syndrome. Br J Psychol 1993; 84: 411-23.

[28] Ray C, Weir WRC, Stewart D, Millar P, Hyde G. Ways of coping with CFS: Development of an illness management questionnaire. Soc Sci Med 1993; 37: 385-91.
[32] Broadbent DE, Cooper PF, Fitzgerald P, Parkes KR. The Cognitive Failures Questionnaire (CFQ) and its correlates. Br J Clin Psychol 1982; 21: 1-16.

[33] Cohen S, Kamarack T, Mermelstein R. A global measure of perceived stress. J Health Soc Behav 1983; 24: 385-96.

[34] Henderson S, Bryne DG, Duncan-Jones P. Neurosis and the Social Environment. Sydney, Australia: Academic Press 1981.

[35] Kanner AD, Coyne JC, Schaefer C, Lazarus RS. Comparison of two modes of stress measurement: Minor daily hassles and uplifts versus major life events. J Behav Med 1981; 4: 1-39.

[36] Fleming JS, Watts WA. The dimensionality of self esteem: Some results from a college sample. J Pers Soc Psychol 1980; 39: 921-9.

[37] Stroop JR. Studies of interference in serial verbal reactions. J Exp Psychol 1935; 18: 643-62.

[38] Erdfelder E, Faul F, Buchner A. GPOWER: A general power analysis program. Behav Res Methods Instrum Comp 1996; 28: 1-11.

[39] O'Carroll RE. The assessment of pre-morbid ability: A critical review. Neurocase 1995; 1: 83-9.

[40] Nathan J, Wilkinson D, Stammers S, Low JL. The role of tests of frontal executive function in the detection of mild dementia. Int J Geriatr Psychiatry 2001; 16: 18-26.

[41] Walsh J, Bedirhan Ustun T. Prevalence and health consequences of insomnia. Sleep 1999; 22: 427-36.

[42] Specchio LM, Prudenzano MP, de Tommaso M, et al. Insomnia, quality of life and psychological features. Brain Res Bull 2004; 63 : 385-91.

[43] Christopher G, Sutherland D, Smith A. Effects of caffeine in nonwithdrawn volunteers. Hum Psychopharmacol 2005; 20: 47-53.

[44] Metzger FA, Denney DR. Perception of cognitive performance in patients with chronic fatigue syndrome. Ann of Behav Med 2002; 24: $106-12$.

[45] Majer M, Welburg LA, Capuron L, Millar AH, Pagnoni G, Reeves WC. Neuropsychological performance in persons with chronic fatigue syndrome: results from a population-based study. Psychosom Med 2008; 70: 829-36.

[46] Thomas M, Sadlier M, Smith A. The effect of multi-convergent therapy on psychopathology, mood and performance of chronic fatigue syndrome patients: A preliminary study. Counselling Psychother Res 2006; 6: 91-9.

[47] Brandt J, Butters N. The neuropsychology of Huntington's Disease. Trends Neurosci 1986; 9: 118-20.

[48] Brown BG, Marsden CD. Cognitive functioning in Parkinson's Disease: from description to theory. Trends Neurosci 1990; 13: 219.

[49] Pham K, Nacher J, Hof PR, McEwens BS. Repeated restrain stress suppresses neurogenesis and induces biphasic PSA-NCAM expression in adult rat dentate gyrus. Eur J Neurosci 2003; 17: 879-86.

[50] Duman RS. Pathophysiology of depression: the concept of synaptic plasticity. Eur Psychiatry 2002; 17: 306-10.

[51] Thomas MA, Smith AP. An investigation of the long term benefits of antidepressant medication in the recovery of patients with chronic fatigue syndrome. Hum Psychopharmacol 2006; 21: 503-9.

[52] Jones JF. An extended concept of altered self: Chronic fatigue and post-infection syndromes. Psychoneuroendocrinology 2008; 33: 119-29.

(C) Thomas and Smith; Licensee Bentham Open.

This is an open access article licensed under the terms of the Creative Commons Attribution Non-Commercial License (http://creativecommons.org/licenses/by-nc/3.0/) which permits unrestricted, non-commercial use, distribution and reproduction in any medium, provided the work is properly cited. 